\title{
Los desafíos de la docencia universitaria
}

\author{
Ana Cecilia Hernández Rodríguez
}

Resumen: Esta ponencia presenta una reflexión acerca de los desafios que enfrenta la docencia universitaria en el momento actual, con respecto a las condiciones mundiales y nacionales.

En el ámbito mundial se analizan brevemente, los problemas relevantes de carácter económico-social, el desarrollo científico y tecnológico, así como la expansión del sistema universitario y los problemas de financiamiento. En el ámbito nacional, se analizan los siguientes aspectos: Vinculación de la Universidad con el sistema educativo nacional, características de la docencia que realizamos, incorporación de la tecnología educativa a la enseñanza en la educación superior, condiciones de los profesores y estudiantes, la administración al servicio de la docencia y las universidades públicas frente a las universidades privadas. A partir de este análisis se expresa la necesidad de revisar, de manera crítica, el quehacer universitario, de recuperar y fortalecer el carácter de "conciencia lúcida”, de buscar, de manera constante la superación académica y de renovar la práctica pedagógica. niveles:

Voy a enfocar este tema, desde dos

- Los desafíos que enfrenta la Universidad como institución, dada las condiciones histórico-sociales dominantes, de carácter mundial y nacional.

- Los desafíos que tiene y debe enfrentar la Educación Superior y la Universidad de Costa Rica de manera particular, con énfasis en los aspectos pedagógicos.

\section{El panorama mundial y su repercusión en nuestro país}

Los cambios tan acelerados que se viven en el ámbito mundial, en diferentes órdenes: económico, político, científico-tecnológico y sociocultural, obligan a la Universidad, a revisar su quehacer de forma integral.

Hoy más que en otras épocas, los universitarios debemos hacer el esfuerzo de ser creativos y de innovar, de lo contrario vamos a perder el papel protagónico que hemos venido manteniendo en el desarrollo de la Ciencia y la Tecnología, en la formación de profesionales de alto nivel y en general en la preparación de los dirigentes. 
Es probable que la Universidad ya haya perdido, en parte esta misión, frente a la investigación que se realiza en institutos especializadas que laboran en función de grandes empresas, así como en relación con procesos de formación y capacitación para atender los procesos específicos de producción o dirección, según requerimiento también de grandes empresas.

Gorostiaga citado por Tünnermann (1998) señala los siguientes problemas como los que están caracterizando a la civilización en el momento actual:

1. La concentración de la riqueza en el $20 \%$ de los habitantes del planeta. El aumento de la riqueza de los multimillonarios.

2. La descapitalización de los países subdesarrollados por fuga de capitales.

3. La concentración del conocimiento en los países desarrollados.

4. Se continúa con altos gastos militares.

5. El tráfico y consumo de drogas.

6. Las crisis y deterioro del medio ambiente.

7. Problemas de empleo para todos los niveles sociales y de escolaridad. Los países del norte también tienen sectores de las poblaciones empobrecidos y marginados, a esto se agrega la reaparición de problemas raciales y culturales.

8. Crisis de gobernabilidad en muchos países (ausencia de liderazgo, ausencia de políticas claras, corrupción).

Ante este panorama y por efecto de la "globalización económica" los países subdesarrollados, como el nuestro, debemos competir, en el campo económico en situaciones adversas y poco claras. No obstante esta competencia debería darse en condiciones de respeto a la dignidad humana, equidad social y solidaridad, tanto en el ámbito mundial como en el nacional, lo cual parece no estar ocurriendo.

Por otra parte, se ha enfocado a la Educación como el medio para resolver muchos de estos problemas "para alcanzar el desarrollo", "para insertarnos en el mercado global", "para resolver múltiples problemas cotidianos", etc., a veces con la distorsión de no considerar otros proyectos y medidas y se generan grandes expectativas, difíciles de alcanzar, máxime si no se cuenta con los recursos humanos (bien preparados y pagados) y los recursos materiales (equipos, infraestructura).

$\mathrm{Al}$ respecto debo aclarar que sí creo en la participación de la Educación en general y de la Educación Superior, para tratar de resolver muchos problemas y mejorar como país en los diferentes campos, sin embargo ésta sola no podrá alcanzar las metas, deben darse programas, medidas, proyectos que definan con claridad políticas de producción y de distribución de la ganancias así como mejorar en cuanto a: recaudación de impuestos, mejorar los controles para evitar los robos de los bienes públicos, agilizar nuestro sistema judicial y buscar alianzas y cooperaciones internacionales que nos fortalezcan.

Otra situación que impacta, de modo directo el trabajo de las universidades, se relaciona con la revolución científica general, en todos los ámbitos del conocimiento, así como la revolución tecnológica, especialmente la relacionada con la informática que ha generado un dinamismo muy fuerte en todos los campos laborales, en el desarrollo del conocimiento y en la educación 
formal y no formal. También podemos mencionar la relacionada con la genética que está produciendo implicaciones muy importantes en el campo de la salud y la ética y próximamente en demografía y otros campos. La Universidad de manera particular no solo debe incorporar toda esta explosión científico-tecnológica sino, ojalá participar de estos procesos, mediante investigación de punta y procesos formativos de gran calidad.

Finalmente otra situación importante se refiere a la expansión y diversificación que ha tenido la Educación Superior, tanto en el ámbito mundial como en el nacional, con cambios importantes como:

- Demanda de nuevos profesores universitarios.

- Diversificación de carreras en contenido y enfoques, diferentes niveles de graduación: pregrado o nivel técnico, grado y posgrado.

- Incremento del número de graduados.

- $\quad$ Problemas de financiamiento.

- $\quad$ Surgimiento de un gran número de instituciones privadas con "Sedes Regionales" en casi todos los cantones del país, que no reciben supervisión o tienen el mínimo de "controles".

Consciente de que solo he enumerado algunos de los problemas relevantes que como planeta y país enfrentamos, cabe preguntarnos: ¿Qué podemos y debemos hacer desde la Universidad?

A continuación analizaré algunos de los problemas y desafíos, con énfasis en la dimensión pedagógica.

\section{Vinculación de la Universidad de Costa Rica con el sistema educativo nacional}

El sistema educativo debe enfocarse como un todo articulado. Lo que suceda en alguno de sus niveles afectará a los otros. La tendencia, en las últimas décadas ha sido darle prioridad a la Educación General Básica, en detrimento de los otros niveles. En Costa Rica, en los últimos años, se ha estado apoyando de manera particular, el nivel de preescolar.

La Educación Secundaria es uno de los niveles más críticos, que requiere ser enfrentado con una visión renovada, con la idea de ofrecer muchas oportunidades, de incorporarle flexibilidad y de que los jóvenes se sientan motivados. El informe Estado de la Nación (1999) indica que los índices de deserción y no incorporación al sistema suman casi el $50 \%$ de los jóvenes que deberían estar en él; también las diferencias significativas que ocurren entre los resultados de colegios públicos y privados en cuanto a las evaluaciones que aplica el Ministerio de Educación Pública. Parte de esta situación afecta a la Educación Superior en cuanto a la preparación de los estudiantes, especialmente quienes provienen de zonas rurales, de colegios nocturnos o de "institutos" que acogen a quienes desertan del sistema formal.

La Universidad no debe permanecer indiferente ante los problemas de la Educación Nacional, debemos proyectarnos y contribuir a solucionar los problemas, por medio de la formación de educadores, de la investigación educativa y de la extensión social. Es importante hacer denuncias en relación con los problemas de equidad de las instituciones educativas, divulgar los resultados de admisión según tipo de colegios y analizarlos según las características 
de cada región y contexto. También es muy importante analizar el papel de las Sedes Regionales en relación con las instituciones educativas deficitarias que se encuentran en su ámbito de influencia.

\section{La docencia que realizamos}

Los universitarios no solamente debemos mirar hacia afuera, sino también hacia adentro, reflexionar acerca de ¿cómo se desarrolla nuestra práctica docente?, ¿qué tipo de educación estamos fomentando? ¿Cuáles son los estilos o tendencias dominantes en la enseñanza y el aprendizaje?

Probablemente las respuestas a estas preguntas nos remitan a considerar que, en un alto grado, mantenemos una tendencia tradicional, cimentada en una posición que asume al conocimiento como una verdad dada, a la enseñanza como "transmisión de contenidos", a la evaluación como devolución de información, al docente como la persona que define, dirige, ejecuta y al estudiante como quien escucha, responde y sigue instrucciones.

En este sentido es urgente cambiar en diversas líneas: Debemos considerar a la educación y a la enseñanza como procesos críticos, de búsqueda, reflexión, indagación y construcción. Los educadores por lo tanto debemos propiciar ambientes apropiados para que estos procesos se den, ambientes caracterizados por la libertad, el respeto y la tolerancia, donde afloren la imaginación, la creatividad, la reflexión y las respuestas innovadoras.

El proceso educativo debe darse en coherencia y clara comprensión del contexto sociocultural, político y económico, inmediato y mediato. Tanto profesores como estudiantes deberán ser investigadores de sus propios procesos de enseñanza y apren- dizaje y de los problemas relevantes vinculados con la disciplina de que se trate.

Aunque el profesor está en una posición de superioridad por su cargo, conocimiento y experiencia, no debe establecer una relación de poder que minimice al estudiante, sino una que favorezca su desarrollo pleno y autonomía. En esta nueva tendencia tanto profesores como estudiantes buscan y trabajan para lograr objetivos comunes, construyen un ambiente "motivador", aprenden y comparten actividades evaluativas; sin que el profesor pierda las condiciones y la autoridad propia del cargo.

\section{Incorporación de la tecnología educativa a la enseñanza en la educación superior}

Continuando con el tema de la enseñanza cabe preguntarse ¿Cuál es el grado de incorporación de la tecnología educativa y en especial de la informática, al desarrollo de la docencia universitaria?

Aunque conozcamos de diversos esfuerzos que se están dando en otras latitudes y aquí mismo, en la preparación de software, de instrumentos, de simulaciones, así como el uso de esta tecnología en la investigación y en la comunicación, al punto de existir universidades virtuales; tenemos que aceptar que la incorporación de la tecnología educativa e informática en la enseñanza universitaria se ha dado lenta y tímidamente. En la Universidad de Costa Rica se han comprado muchas computadoras para apoyar los procesos administrativos, muchas para apoyar los procesos de investigación y de comunicación y muy pocas para apoyar la docencia de manera directa.

Tanto autoridades como docentes debemos hacer un esfuerzo por incorporar esta tecnología y aprovechar sus ventajas 
para: modernizar, flexibilizar y dinamizar los procesos de enseñanza y aprendizaje y para lograr un mejor aprovechamiento del tiempo, con el propósito de mejorar la calidad de la docencia y de facilitar el acceso al conocimiento a todos los estudiantes y no de abaratar costos.

El uso de esta tecnología debe darse dentro de una perspectiva crítica, que le permita al estudiante indagar, profundizar, reflexionar, crear, proponer y construir, que favorezca el aprendizaje significativo y no dentro de un enfoque tradicional (conductista) donde solo siga instrucciones y se contesten preguntas. También esta tecnología nos debe permitir un contacto rápido y directo con egresados y la comunidad en general y organizar procesos de actualización, en el marco de una Educación Permanente.

\section{Estudiantes y profesores}

Siempre ubicados en los procesos de enseñanza y aprendizaje, un aspecto que se ha convertido en un desafío es el de los estudiantes y los profesores.

En el siglo XX, en relación con la Educación ocurrieron dos procesos muy importantes en nuestro país, la expansión de la Enseñanza Secundaria, a mediados de siglo y la expansión de la Educación Superior en las últimas tres décadas. Éstas, entre otras situaciones, han hecho cambiar a profesores y estudiantes. Se ha debido contratar a muchos profesores, el estereotipo del profesor "maduro" de saco y corbata o de gabacha, de décadas anteriores, ha variado. Podríamos afirmar que quizás asistimos a una brecha generacional entre académicos, en la Universidad de Costa Rica, que se refleja en: si son profesores interinos o en propiedad, en la jornada si son de tiempo completo o tiempo parcial, en los salarios que reciben; aunque éstos, han perdido su valor real en general, debemos reconocer que los profesores que han ingresado a la Universidad recientemente, reciben salarios muy bajos; también se refleja en enfoques y prácticas que no vamos a analizar en este momento.

En cuanto a los estudiantes escuchamos la queja constante de que cada vez, vienen más mal preparados, que no razonan, que tienen pocos conocimientos, que no tienen hábitos de estudio, que no están motivados, etc., etc. $\mathrm{Al}$ respecto debemos analizar, en primer lugar, la heterogeneidad de los colegios de donde proceden y como institución, plantear opciones para nivelarlos y apoyar a los colegios deficitarios. No podemos convertirnos en una Universidad que funciona con base en "la medida" de los colegios privados del área metropolitana, esto sería caer en un darwinismo cultural como lo denominan Ayuste y otros (1994).

También considerar la heterogeneidad en edad que encontramos hasta en los cursos de Estudios Generales.

Por otra parte nos debemos preguntar cómo es el "estudiante posmoderno" que recibimos: es el estudiante de la imagen y quizás menos el estudiante lector, "el estudiante de las pantallas", de la pantalla de la T.V., de la computadora, del vídeo-juego, del cine... y quizás menos el estudiante que dialoga, que discute, que comparte en grupo. Pienso que este es un aspecto que debemos investigar con profundidad.

\section{La administración al servicio de la academia}

Quizás debido al crecimiento de las universidades y a algunas prácticas administrativas, hemos ido evolucionando hacia procesos administrativos que han perdido el norte: el estar al servicio de la 
academia, la docencia y de los estudiantes de manera especial.

La administración parece haberse convertido en un fin en sí misma y se dan entonces situaciones que deben corregirse:

- $\quad$ Existe una distancia importante entre los estudiantes y docentes y las autoridades universitarias, en todos los niveles. En algunas oficinas hay secretarias o asesores entrenados para no permitir el acceso a estas autoridades.

- $\quad$ Para tratar de que se atiendan problemas académicos, hay que hacer antesala de largas horas, valerse de amigos allegados a las autoridades, hay que presentar múltiples justificaciones, etc.

- Los trámites son muy lentos, muchas veces pasan de oficina en oficina, algunas veces se extravían, se necesitan muchos vistos buenos.

- No siempre usamos la información existente en la misma universidad para resolver los problemas, por ejemplo ¿Cómo se hace el "congelamiento" y "descongelamiento" de plazas? Como respuesta a comportamientos de matrícula y número de graduados, de proyectos de investigación o de acción social, o con base en los criterios del director o la directora que más insiste, mejor justifica o es el más conocido.

- Pareciera que tampoco hemos ganado en agilidad administrativa y en eficiencia con la incorporación de la tecnología informática, por lo menos no se aprecian cambios significativos en procesos como la matrícula, los nombramientos y la atención general a estudiantes y profesores.

\section{Las universidades públicas frente a las universidades privadas}

El proceso de expansión de la Educación Superior, antes mencionado, unido a tendencias neoliberales como "competitividad" y "libre empresa" han influido en la proliferación de universidades privadas en nuestro país y en otros.

En Costa Rica la situación ya rima con el caos y el ridículo. Existen 48 universidades privadas, algunas de las cuales funcionan sin autorización y en otras no hay claridad acerca de si todas las carreras están autorizadas. Con respecto a estas irregularidades me interesa plantear los siguientes aspectos:

- La universidad privada de por sí no es mala; lo malo es que en nuestro país no podemos garantizar su calidad, porque no hemos aplicado controles claros, muchas funcionan con fines de lucro y algunas podrían ser verdaderos fraudes sociales.

- La acreditación mediante el Sistema Nacional de Acreditación de la Educación Superior (SINAES) se propone como alternativa para intentar poner orden y garantizar programas con una calidad aceptable. Esperamos que este procedimiento funcione y no sea solo un modo para bendecir la situación actual.

- $\quad$ Otro aspecto que debe considerarse es la cantidad de graduados en la mayoría de los campos, que no encuentran trabajo, fundamentalmente los de las instituciones privadas, porque en la mayoría de los cambios parece que los empleadores siguen prefiriendo a los graduados de las universidades públicas. 
- ¿Cuál ha sido el papel de las universidades públicas y de la Universidad de Costa Rica, de manera particular, frente a esta situación de las universidades privadas?

Mi impresión es que se ha combinado la indiferencia con la complicidad, porque en la práctica no hemos hecho casi nada, hemos dejado que el caos crezca a pesar de conocer muchas irregularidades y en muchas ocasiones se han constituido en una "extrita" y algunos profesores hasta se han matriculado en alguno de sus programas de grado o posgrado, aunque conozcan que en estas universidades no se realizan investigaciones para fundamentar los estudios de posgrado.

Considero que es muy importante que, en este momento, en que se han presentado algunas denuncias con respecto a estas instituciones que, en el ámbito de CONARE o de la Universidad de Costa Rica, se constituyan múltiples comisiones para estudiar la ley y el reglamento que regulan las actividades del CONESUP y las condiciones que deben tener las universidades privadas para que se garantice una calidad aceptable; hacer una propuesta de nueva ley y reglamento; definir las condiciones que debe tener una oficina de apoyo al CONESUP que podrían financiar las propias universidades privadas, para realizar un trabajo de supervisión objetivo y técnico. Podría también analizarse la propaganda que realizan estas universidades, que se da como si vendieran cualquier mercancía y orientarla hacia brindar información y no "embarcar" a jóvenes y a adultos a realizar estudios en condiciones poco claras.

- Finalmente, ante esta explosión de universidades privadas con sedes en todos los pueblos, debemos en las universidades públicas mirarnos hacia adentro y con toda responsabilidad analizar qué nos ha sucedido en cuanto a la adaptación y las respuestas que hemos venido dando a las nuevas condiciones y demandas de la sociedad costarricense (no sólo de los grupos de empresarios); preguntarnos, ¿de qué manera favorecemos al estudiante que trabaja?, ¿de qué manera llegamos a las comunidades más elejadas?, ¿cómo funcionan y cómo se han venido fortaleciendo las sedes regionales?. Quizás CONARE debería transformarse en un verdadero sistema universitario, con más acercamiento entre las universidades públicas, generar más fluidez en los procesos de reconocimiento de estudios en éstas y con una verdadera coordinación en cuanto a la oferta de especialidades y la cobertura de las demandas en todo el país. También debemos analizar nuestra capacidad de respuesta ante los procesos de innovación educativa y aceptar que también este proceso de explosión de universidades privadas, nos está diciendo algo acerca de la inercia de nuestro funcionamiento general.

\section{A manera de síntesis}

"El cambio de época" del que muchos hablan, nos debe llevar a revisar y reflexionar los diferentes ámbitos de quehacer humano y uno de los más importantes es el educativo y en él, el de la Educación Superior. Es conveniente analizar, con una visión holística, la pertinencia, los aportes y la calidad de su labor.

La universidad pública y, de manera específica, la Universidad de Costa Rica debe reflexionar su quehacer considerando, desde el papel que juega en el estudio de diversos problemas económicos, sociales, técnicos y políticos y la asesoría que brinda o puede brindar ante múltiples problemas sociales y técnicos, y hasta la 
cantidad y calidad de sus graduados. Actuar con sentido crítico, recuperar o reforzar su papel de conciencia lúcida.

Un esfuerzo y meta constante debe ser la búsqueda de la excelencia académica y el mejoramiento de todo lo que esto implica. Un punto fundamental es el que se refiere a sus profesores, es necesario exigir y a la vez facilitar las condiciones requeridas para su superación constante, tanto en el área de especialidad como en el campo pedagógico. También es necesario revisar el "régimen académico" y los salarios. Tanto profesores como estudiantes deben desarrollar las destrezas del autoaprendizaje, la formación permanente y la metacognición.

La Universidad debe revisar sus políticas de admisión, mantener una posición de apertura democrática hacia los estudiantes de todas las clases sociales y regiones y ser una instancia de homogenización, acercamiento y movilidad social. En este sentido, la posición y el papel de las sedes regionales es determinante, debemos fortalecerlas en todos los ámbitos y atender las demandas que diversas comunidades nos planteen, por medio de la apertura de recintos y aulas universitarias. Debemos también recurrir a las posibilidades que nos brinda la tecnología para flexibilizar y ampliar nuestras ofertas, tanto en grado como en posgrado.
Para atender y tratar de solucionar los múltiples problemas y desafíos de la docencia universitaria debemos recurrir a la investigación, la creatividad, la imaginación y el compromiso de todos.

\section{Referencias bibliográficas}

Ayuste, Ana; Flecha, Ramón; López Palma, Fernando y Lleras, Jordi. Planteamientos de la Pedagogía Crítica. Barcelona: Editorial Graó, de Serveis Pedagógics. 1994.

Freire, Paulo. Pedagogía de la autonomía. Saberes necesarios para la práctica educativa. México, D.F., siglo XXI editores, S. A. 1997.

Proyecto Estado de la Nación. Estudios de la Nación en Desarrollo Humano sostenible: un análisis amplio y objetivo sobre la Costa Rica que tenemos a partir de los indicadores más actuales 1998. 1era. edición. San José, costa Rica; Proyecto Estado de la Nación. 1999.

Tünnermann B., Carlos. La transformación de la Educación Superior: retos $y$ perspectivas. Heredia, Costa Rica: EUNA. 1998. 